\title{
An Unusual Case of a Pulmonary-Renal Syndrome with Negative Anti-GBM and ANCA Antibodies
}

\section{Fabrício Malaguez Webber ${ }^{1 *}$, Edson Ricardo Junior ${ }^{2}$, Maria Belen López Morillo', Emanuel Malaguez Webber ${ }^{3}$}

${ }^{1}$ Centro de Especialidades Médicas San Lorenzo, El Salvador, Chile

${ }^{2}$ Hospital Antônio Prudente, Natal, Brazil

${ }^{3}$ Hospital Regional Teresinha Gaio Basso, São Miguel do Oeste, Brazil

Email: *FabricioWebber@doctor.com

How to cite this paper: Webber, F.M., Ricardo Junior, E., Morillo, M.B.L. and Webber, E.M. (2018) An Unusual Case of a Pulmonary-Renal Syndrome with Negative Anti-GBM and ANCA Antibodies. Case Reports in Clinical Medicine, 7, 461-469. https://doi.org/10.4236/crcm.2018.78040

Received: July 13, 2018

Accepted: August 6, 2018

Published: August 9, 2018

Copyright $\odot 2018$ by authors and Scientific Research Publishing Inc. This work is licensed under the Creative Commons Attribution International License (CC BY 4.0).

http://creativecommons.org/licenses/by/4.0/

\begin{abstract}
Pulmonary renal syndrome (PRS) is a high mortality, rare disorder presenting with diffuse alveolar hemorrhage and progressive acute glomerulonephritis. This syndrome is often caused by autoimmune entities, the most frequent being ANCA positive vasculitis and anti-GBM disease. We report a case of a 34-year-old Chilean woman, who initially presents with anemia and after a few days of inpatient management, starts with progressively worsening dyspnea, decrease in renal function and hematuria. The patient is initially diagnosed with pneumonia, but further evaluation using Thorax CT scan and renal biopsy confirms the suspicion of PRS. The case is of particular interest due to the lack of extensive bibliography on anti-GBM and ANCA negative pulmonary-renal syndrome, an uncategorized subtype of this syndrome with unknown optimal management.
\end{abstract}

\section{Keywords}

Pulmonary Renal Syndrome, Goodpasture, ANCA, Anti-GBM

\section{Introduction}

The Pulmonary-Renal Syndrome (PRS) is a rare syndrome caused by a variety of etiologies. The patient presentation is often confused with pneumonia. However, a proper diagnosis can be achieved if there is a high degree of suspicion by the clinician, with major etiologies being anti-neutrophil cytoplasmic antibody (ANCA) vasculitides and anti-glomerular basement membrane (GBM) disease, accounting for up to $75 \%$ of PRS cases in intensive care units (ICU) [1]. That 
said, a lesser known and studied subset of PRS may be caused by Systemic Lupus Erythematosus (SLE), cryoglobulinemias and drugs, leading to an even greater diagnosis challenge [2]. The case we present is accompanied by negative ANCA and anti-GBM antibodies. We find it relevant to report this case as we aim to discuss the contradictory findings during this patient workup, while evaluating other causes of PRS, and suggesting a treatment rationale along with a possible novel immunological target for diagnosis.

\section{Case Report}

A 34-year-old Chilean female presented to our emergency service $(04 / 02 / 2009)$ with a chief complaint of increasingly progressive dyspnea and fatigability associated with two episodes of bloody stool and vaginal bleeding. Physical exam shows conjunctival pallor and palpable liver edge with tenderness. No jaundice is observed. Laboratory results revealed anemia (Hemoglobin: $6.3 \mathrm{~g} / \mathrm{dL}$ ) and prothrombin time of $35.9 \%$. The patient is transferred to the inpatient unit for transfusional therapy and further study of her anemia and vaginal bleeding. On the following day, physical exam remains unchanged, gynecological consult is obtained and endometrial disease is discarded as the cause of her bleeding, abdominal ultrasound shows splenomegaly and suggestive signs of liver damage. The patient is kept under inpatient setting for proper correction of the underlying hematological disorders. During the night of the fifth day of admission, the patient presents with chest pain and progressive dyspnea with $\mathrm{SpO}_{2} \%$ of $75 \%$. The patient is sent to the regional hospital for admission to the ICU due to acute respiratory failure and endovenous ceftriaxone is initiated due to suspected pneumonia. Thoracic computed tomography shows a clear interstitial pattern while laboratory exams show an increase in creatinine levels $(2.87 \mathrm{mg} / \mathrm{dL})$ and macroscopic hematuria. A pulmonary-renal syndrome is suspected and methylprednisolone is initiated along with mechanical ventilation due to worsening respiratory failure, with progressively decreasing $\mathrm{PaO}_{2} / \mathrm{FiO}_{2}$, getting as low as 79 $\mathrm{mmHg}$. The patient is transferred to a higher complexity hospital in Santiago via air ambulance. On arrival, patient $\mathrm{SpO}_{2}$ is $55 \%$ and orotracheal tube aspiration shows multiple blood clots.

Bronchoalveolar lavage is performed and diffuse alveolar hemorrhage is confirmed, while samples were submitted for the following microbiological tests: Gram stain and bacterial cultures; Kinyoun stain and mycobacterial cultures; Calcofluor-white stain and fungi cultures; polymerase chain reaction for $P$. jiroveci; respiratory viral panel. All the performed microbiological tests were negative.

Rheumatologic studies results show the following: ANA 1/320, normal C3 levels, low $\mathrm{C} 4$ levels, anti-Ro positive and Anti-La positive. Anti-DNA negative, anti-DNA (Farr essay) negative, PR3-ANCA negative, MPO-ANCA negative, Anti-GBM negative and antiphospholipids negatives. Coombs test is positive with low haptoglobin. 
Figure 1 allows for a summary of the clinical evolution and interventions performed.

Patient alveolar hemorrhage was successfully treated with methylprednisolone pulses (3 grams), plasmapheresis (10 sessions) and two pulses of cyclophosphamide $500 \mathrm{mg}$. However, despite pulmonary improvement, during ICU stay the patient progressed with a persistent decrease in hematocrit and platelets, being interpreted as hemolytic anemia, thus being treated with two additional methylprednisolone pulses (totaling 5 grams). After initial stabilization, the patient presented with melena and a further decrease in hematocrit with normal LDH and haptoglobin. Upper gastrointestinal endoscopy and further analysis were compatible with upper gastrointestinal bleeding due to portal hypertensive gastropathy. There was also an inappropriate bone marrow response to the anemia with normal-to-low erythropoietin levels, requiring its administration, which was attributed to antibiotics and the concurrent systemic inflammation.

Renal biopsy was positive for crescentic pauci-immune glomerulonephritis with $35 \%$ presence of tubular atrophy and interstitial fibrosis. The rapid progression of the renal failure was managed with transitory dialysis, allowing to keep stationary creatinine levels around 1.2 to $1.4 \mathrm{mg} / \mathrm{dL}$.

Even though the patient was under evaluation for possible autoimmune hepatitis, no autoimmune hepatitis markers were found elevated during hospitalization; Anti-LKM, Anti-AMA, ASMA and anti-tissue transglutaminase were all found to be negative. Antibodies against viral hepatitis were also found to be negative.

The last rheumatological markers before discharge and their levels in the following years are summarized in Table 1 . Worthy of notice was the normalization of Anti-La antibodies $(10.8 \mathrm{U} / \mathrm{mL}$, reference range 0 - 20) in December/2010, while keeping a slightly elevated Anti-Ro antibody that normalized by May/2011 (from 22.8 to $18.1 \mathrm{U} / \mathrm{mL}$, reference range 0 - 20).

The patient maintained a stable regimen with prednisolone $8 \mathrm{mg} / \mathrm{day}$, hydroxychloroquine $200 \mathrm{mg} /$ day and mycophenolate 2 grams/day, not presenting further disease exacerbations or loss of renal function until May/2016, when the patient is evaluated for chronic diarrhea. Mycophenolate was removed due to gastrointestinal side effects, and chronic diarrhea subsides with relative normalization of renal function to previous levels. Hydroxychloroquine use had to be discontinued due to the appearance of retinopathy.

The patient, up to the date of publication, has not had any new PRS crisis. However, her renal failure has continued to progress and the patient is currently on peritoneal dialysis due to end-stage-renal-disease (ESRD). A trial of Rituximab was administred on two occasions, yielding no improvement of renal function and causing acute avascular necrosis of the patella during the two infusions. Currently, the patient's only immunomodulatory therapy is methylprednisolone $8 \mathrm{mg} /$ day as other therapies have produced adverse events or are contraindicated due to ESRD. 


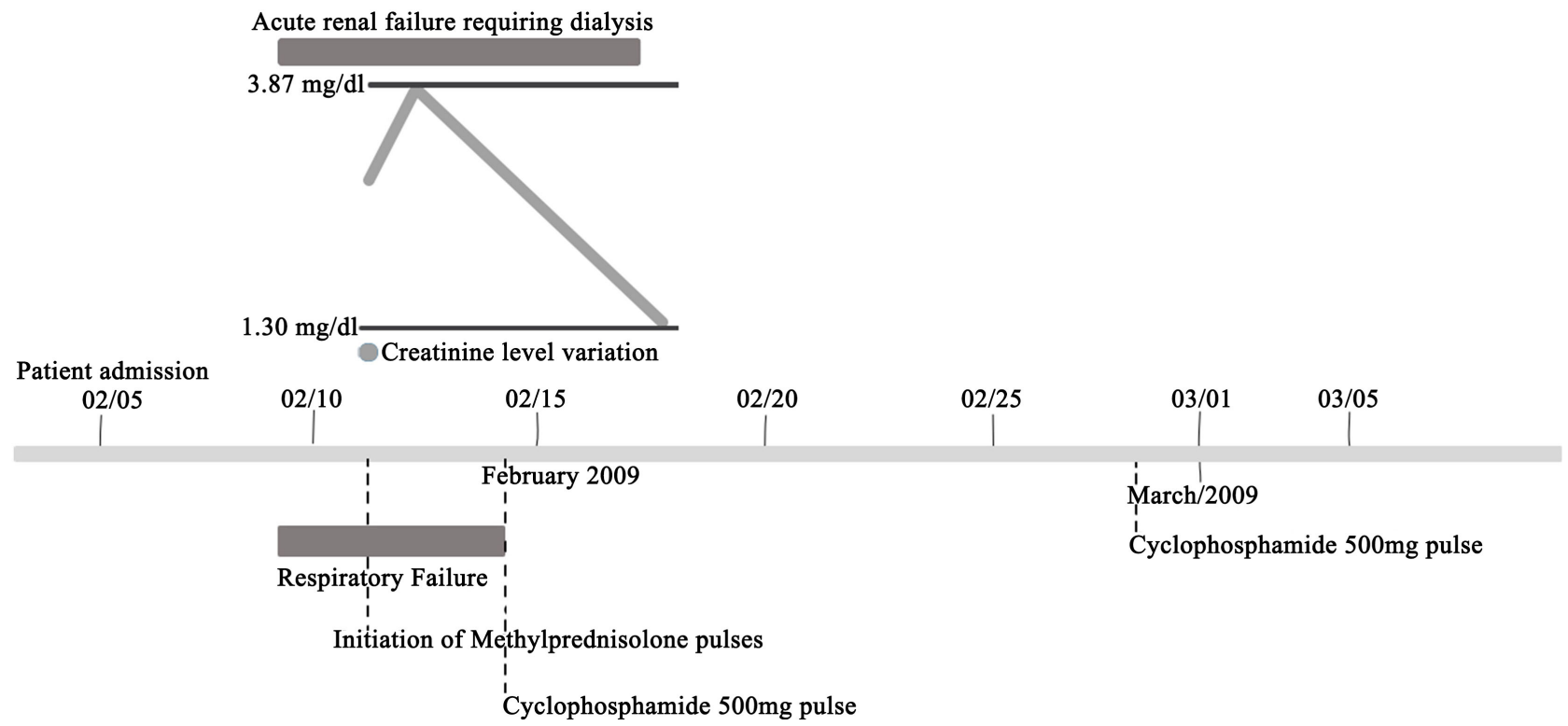

Figure 1. Timeline of patient clinical during hospitalization.

Table 1. Patient serological progression over time.

\begin{tabular}{ccc}
\hline Antibody essay & Result at discharge & Result on follow-up \\
\hline ANA (IFI) & $1 / 320$ & $1 / 320$ \\
Anti B2 glycoprotein & Negative & Negative \\
Anti Ro & Positive & Positive, then negativization \\
Anti La & Positive & Negative \\
Anti RNP & Negative & Negative \\
Anti Smith & Negative & Negative \\
Anti-DNA & Negative & Negative \\
Cryoglobulins & Negative & Negative \\
Antimitochondrial antibodies & Negative & Negative \\
Anti Ribosomal P Protein & Negative & Negative \\
Anti Smooth Muscle Antibody & Negative & Negative \\
Antiphospholipids & Negative & Negative \\
Lupic anticoagulant & Negative & Negative \\
Antineutrophil Cytoplasmic & Negative & Negative \\
Antibody (MPO and PR3) & Negative & Negative \\
Anti glomerular basal membrane & & \\
\hline & &
\end{tabular}

\section{Discussion}

PRS is a rare disorder caused by heterogeneous pathological entities with the common symptom presentation of diffuse alveolar hemorrhage and progressive acute glomerulonephritis. The etiologies for such syndrome are diverse but usually derive from an autoimmune vascular insult with inflammation and ne- 
crosis of blood vessels and the glomeruli, ranging from the most common causal agents such as ANCA positive small vessel vasculitis (such as granulomatosis with polyangiitis, microscopic polyangiitis), anti-GBM disease and systemic erythematous lupus to less frequent causative agents, such as drug-induced vasculitis (e.g., penicillamine, propylthiouracil), mixed cryoglobulinemia and the idiopathic form, to name a few [2].

The PRS has a high mortality rate, which seems to present in a bimodal fashion; During the acute phase a catastrophic clinical debut can present as diffuse alveolar hemorrhage causing airway obstruction and sudden onset of acute respiratory insufficiency. Even more, due to the low frequency of the disease, it can be inadvertently misdiagnosed as pneumonia and delay appropriate therapy, as some patients may present only with pulmonary infiltrates, dyspnea and fever.

The increased mortality in the chronic phase of the disorder could be attributed to potential adverse effects of the therapy, a progressively declining renal function or, even the management of the complex multisystemic disorders underlying this syndrome [3].

No current clinical criteria or score has been developed for rapidly assessing the probability of PRS. Papiris et al. (2007) have suggested considering this diagnosis in the presence of pulmonary infiltrates along with following findings: hemoglobin decrease, rapidly progressing renal failure, sinusitis, mononeuritis multiplex, polyarthralgia, severe asthma attack, pericarditis, cerebral ischemia, purpura or congestive heart failure [1]. We suggest that further suspicion should be raised in the presence of any immunological marker or personal history of autoimmune diseases.

Once a syndromic diagnosis is established, the question of the underlying etiology remains unresolved, which we try to explore next.

Systemic Erythematous Lupus (SLE) is one cause of PRS with the frequency of such presentation being as low as $2 \%$ in SLE patients. However, this clinical presentation is rarely the first manifestation of the disease [4]. More importantly, the presence of pauci-immune glomerulonephritis in the context of an SLE associated PRS would be, although possible [5] [6], a very unlikely presentation of the disease, as the SLE glomerular disease commonly occurs as a result of a type III hypersensitivity with antibody-deposition being the primary pathogenic insult [7]. Even though these two findings are suggestive of another etiology for the PRS presented in our patient, we find it relevant to point out that this patient fills the Systemic Lupus Collaborating Clinics (SLICC) criteria for SLE [8] (Table 2) and it is worthwhile to notice the transitory elevation of the Anti-Ro antibody which also points towards the possible SLE diagnosis.

Another possibility that has been explored was the Mixed Connective-Tissue Disease (MCTD), due to its extensive clinical presentations including alveolar hemorrhage [9]. Although a possibility, MCTD is not a likely diagnosis for our patient, as she lacks the hallmark positive anti-RNP findings, required in both Alarcon-Segovia and Kahn's criteria, currently the most accurate criteria for 
Table 2. Patient presentation and criteria met according to ACR SLE criteria and the newer SLICC criteria. SLICC criteria requires the presence of 4 of the findings, being at least one immunological and one clinical. It is also noteworthy that this criterion mandates that if the patient presents two highly correlated findings, such a positive Coombs, and hemolytic anemia, only one of these findings should be counted [8].

\begin{tabular}{|c|c|c|c|}
\hline \multicolumn{4}{|c|}{ Patient presentation according to different SLE criteria } \\
\hline \multicolumn{2}{|l|}{ SLICC Criteria } & \multicolumn{2}{|c|}{$\begin{array}{l}\text { American College of Rheumatology } \\
\text { (ACR) SLE criteria }\end{array}$} \\
\hline Acute Cutaneous Lupus & $x$ & Malar Rash & $\times$ \\
\hline Chronic Cutaneous Lupus & $x$ & Discoid Rash & $\times$ \\
\hline Oral/Nasal Ulcers & $x$ & Photosensitivity & $x$ \\
\hline Non-scarring alopecia & $x$ & Oral Ulcers & $x$ \\
\hline Arthritis & $x$ & Nonerosive Arthritis & $x$ \\
\hline Serositis & $x$ & Serositis & $x$ \\
\hline Renal & $\sqrt{ }$ & Renal & $\sqrt{ }$ \\
\hline Neurologic & $\times$ & Neurologic & $x$ \\
\hline Hemolytic anemia & $\sqrt{ }$ & Hematologic & $\sqrt{ }$ \\
\hline Leukopenia & $\times$ & & \\
\hline Thrombocytopenia & $\sqrt{ }$ & & \\
\hline ANA & $\sqrt{ }$ & Immunologic & $\times$ \\
\hline Anti-DNA & $\times$ & ANA & $\sqrt{ }$ \\
\hline Anti-Sm & $\times$ & & \\
\hline Antiphospholipid Ab & $\times$ & & \\
\hline Low complement $\left(\mathrm{C}_{3}, \mathrm{C}_{4}, \mathrm{CH}_{50}\right)$ & $\sqrt{ }$ & & \\
\hline Direct Coombs test & $x$ & & \\
\hline
\end{tabular}

MCTD [10]. Moreover, the presence of renal complications which, even though they have been described, are uncommon in MCTD [11]. Common MCTD clinical features (including the ones used as diagnostic criteria), such as hand edema, synovitis, myositis, Raynaud phenomenon, and acrosclerosis, were all absent in the course of patient's disease. Thus, other differential diagnoses ought to be investigated.

Finally, other important etiologies to be considered in a patient with PRS are the ANCA-positive vasculitides. The ANCA-positive vasculitides are conformed by three major clinical syndromes; microscopic polyangiitis (MPA), Churg-Strauss syndrome and Granulomatosis with Polyangiitis (GPA). As the name suggests, in these three etiologies, there is a well-characterized role of ANCA antibodies, specifically, antibodies directed against myeloperoxidase (MPO) and proteinase 3 (PR3) proteins in neutrophils cytoplasm, in the pathogenesis of these diseases and the expression of PRS [12].

Although it is possible to have GPA with negative ANCA antibodies, this unusual presentation is usually associated with a decreased likelihood of renal 
compromise [13]. Meanwhile, the lack of eosinophilia in the absence of use of glucocorticoids in the initial laboratory findings, as the lack of asthma-like symptoms, steer the diagnosis away from a possible Churg-Strauss syndrome. Lastly, the low $\mathrm{C} 4$ complement levels make the MPA diagnosis, as well as other vasculitides, less likely since low $\mathrm{C} 4$ levels would be expected in non-ANCA related disorders, as ANCA-positive vasculitides obey an alternative pathway activation [14] with increased to normal C4 levels [15] physiopathogenesis.

Thus, due to the lack of supportive findings towards the previously mentioned etiologies, we classified our patient entity as undefined PRS. While most of the patients in this category may have other described etiologies, the contradictory findings of our case and lack of serological markers led us to further search for possible novel immunological markers. Not tested in this case, and a possible marker for undefined pulmonary-renal syndrome is the anti-endothelial cell antibodies, shown to be linked to ANCA-negative pauciimune glomerulonephritis [16] [17], propylthiouracil-induced ANCA positive vasculitis [18] and antibody-mediated microvascular insult in idiopathic pulmonary fibrosis [19]. Further studies are required to explore this possible marker in this subset of PRS patients.

From the treatment standpoint, once the undefined PRS diagnosis has been achieved, due to the high mortality rate of this syndrome [3], treatment must be initiated as soon as possible. A review in the current literature yields reports in Japan [20], Germany [21], United States [22] with interesting similarities, and an american series report of patients with diffuse alveolar hemorrhage (20 patients), where one of them presented with ANCA negative serology [23], relevant to our case. Common to all these and our case is the improvement of the patient condition with the use of plasmapheresis and immunosuppression, both by corticosteroids pulses only or combined with cyclophosphamide pulses. Stemming from these case reports outcomes, our own experience, and based on the rationale of the autoimmune origin of these insults, a more aggressive therapeutic regimen based on early plasmapheresis, corticosteroid pulses and cyclophosphamide pulses seems to be an appropriate therapy for this subset of PRS patients.

\section{Conclusion}

The PRS, being a rare disease, presents itself as a significant diagnostic challenge to the clinician. Even when there is clinical suspicion of this syndrome, less common etiologies may present and generate further doubt regarding the diagnosis and proper patient work-up. ANCA positive vasculitides and anti-GBM disease are responsible for the majority of the PRS in ICUs [1]. The data regarding undefined PRS presenting negative ANCA and anti-GBM negative serology is limited. However, we suggest based on similar reports that the approach of combining corticosteroids and cyclophosphamide pulses, along with plasmapheresis, to be considered as a therapeutic strategy. Further studies are yet required to explore novel immunological markers, therapeutic options and proto- 
cols in this subset of PRS patients, hopefully shedding light for physicians facing a high mortality disease where the lack of expected immunological findings may delay this difficult diagnosis.

\section{Consent}

Written informed consent was obtained from the patient for publication of this case report.

\section{Conflict of Interests}

No conflict of interests are reported by any of the authors.

\section{References}

[1] Papiris, S., Manali, E., Kalomenidis, I., Kapotsis, G., Karakatsani, A. and Roussos, C. (2007) Bench-to-Bedside Review: Pulmonary-Renal Syndromes-An Update for the Intensivist. Critical Care, 11, 213. https://doi.org/10.1186/cc5778

[2] Mccabe, C., Jones, Q., Nikolopoulou, A., Wathen, C. and Luqmani, R. (2011) Pulmonary-Renal Syndromes: An Update for Respiratory Physicians. Respiratory Medicine, 105, 1413-1421. https://doi.org/10.1016/j.rmed.2011.05.012

[3] Gallagher, H., Kwan, J.T.C. and Jayne, D.R.W. (2002) Pulmonary Renal Syndrome: A 4-Year, Single-Center Experience. American Journal of Kidney Diseases, 39, 42-47. https://doi.org/10.1053/ajkd.2002.29876

[4] Keane, M. and Lynch, J. (2000) Pleuropulmonary Manifestations of Systemic Lupus Erythematosus. Thorax, 55, 159-166. https://doi.org/10.1136/thorax.55.2.159

[5] Uskudar Cansu, D., Temiz, G., Acikalin, M. and Korkmaz, C. (2017) Pauci-Immune Lupus Nephritis: Possibility or Co-Incidence? European Journal of Rheumatology, 4, 73-75. https://doi.org/10.5152/eurjrheum.2016.013

[6] Fayaz, A., Pirson, Y., Cosyns, J., Yango, J. and Lambert, M. (2008) Pauci-Immune Necrotizing and Crescentic Glomerulonephritis in a Patient with Systemic Lupus Erythematosus. Clinical Nephrology, 69, 290-293. https://doi.org/10.5414/CNP69290

[7] Lech, M. and Anders, H. (2013) The Pathogenesis of Lupus Nephritis. Journal of the American Society of Nephrology, 24, 1357-1366. https://doi.org/10.1681/ASN.2013010026

[8] Petri, M., Orbai, A.-M., Alarcon, G.S., et al. (2012) Derivation and Validation of the Systemic Lupus International Collaborating Clinics Classification Criteria for Systemic Lupus Erythematosus. Arthritis \& Rheumatism, 64, 2677-2686. https://doi.org/10.1002/art.34473

[9] Bull, T., Fagan, K. and Badesch, D. (2005) Pulmonary Vascular Manifestations of Mixed Connective Tissue Disease. Rheumatic Disease Clinics of North America, 31, 451-464. https://doi.org/10.1016/j.rdc.2005.04.010

[10] Alarcón-Segovia, D. and Cardiel, M. (1989) Comparison between 3 Diagnostic Criteria for Mixed Connective Tissue Disease. Study of 593 Patients. The Journal of Rheumatology, 16, 328-34.

[11] Lundberg, I. (2005) The Prognosis of Mixed Connective Tissue Disease. Rheumatic Disease Clinics of North America, 31, 535-547.

https://doi.org/10.1016/j.rdc.2005.04.005 
[12] Jennette, J. (2006) Pathogenesis of Vascular Inflammation by Anti-Neutrophil Cytoplasmic Antibodies. Journal of the American Society of Nephrology, 17, 1235-1242. https://doi.org/10.1681/ASN.2005101048

[13] Miloslavsky, E., Lu, N., Unizony, S., et al. (2016) Myeloperoxidase-Antineutrophil Cytoplasmic Antibody (ANCA)-Positive and ANCA-Negative Patients with Granulomatosis with Polyangiitis (Wegener's): Distinct Patient Subsets. Arthritis \& Rheumatology, 68, 2945-2952. https://doi.org/10.1002/art.39812

[14] Xiao, H., Schreiber, A., Heeringa, P., Falk, R. and Jennette, J. (2007) Alternative Complement Pathway in the Pathogenesis of Disease Mediated by Anti-Neutrophil Cytoplasmic Autoantibodies. The American Journal of Pathology, 170, 52-64. https://doi.org/10.2353/ajpath.2007.060573

[15] Deshayes, S., Aouba, A., Khoy, K., Mariotte, D., Lobbedez, T. and Martin Silva, N. (2018) Hypocomplementemia Is Associated with Worse Renal Survival in ANCA-Positive Granulomatosis with Polyangiitis and Microscopic Polyangiitis. PLoS ONE, 13, e0195680. https://doi.org/10.1371/journal.pone.0195680

[16] Sampathkumar, K., Ramakrishnan, M., Sah, A., Gowtham, S. and Ajeshkumar, R. (2010) ANCA Negative Pauci-Immune Glomerulonephritis with Systemic Involvement. Indian Journal of Nephrology, 20, 43.

https://doi.org/10.4103/0971-4065.62096

[17] Cong, M., Chen, M., Zhang, J., Hu, Z. and Zhao, M. (2008) Anti-Endothelial Cell Antibodies in Antineutrophil Cytoplasmic Antibodies Negative Pauci-Immune Crescentic Glomerulonephritis. Nephrology, 13, 228-234. https://doi.org/10.1111/j.1440-1797.2007.00894.x

[18] Yu, F., Zhao, M., Zhang, Y., Zhang, Y. and Wang, H. (2005) Anti-Endothelial Cell Antibodies (AECA) in Patients with Propylthiouracil (PTU)-Induced ANCA Positive Vasculitis Are Associated with Disease Activity. Clinical \& Experimental Immunology, 139, 569-574. https://doi.org/10.1111/j.1365-2249.2005.02725.x

[19] Magro, C., Waldman, W., Knight, D., et al. (2006) Idiopathic Pulmonary Fibrosis Related to Endothelial Injury and Antiendothelial Cell Antibodies. Human Immunology, 67, 284-297. https://doi.org/10.1016/j.humimm.2006.02.026

[20] Yamaguchi, H., Shirakami, A., Haku, T., et al. (2013) Pulmonary-Renal Syndrome with Negative ANCAs and Anti-GBM Antibody. Case Reports in Nephrology, 2013, Article ID: 434531. https://doi.org/10.1155/2013/434531

[21] Fröhlich-Gildhoff, M., Jabs, W., Berhold, C., et al. (2016) A Rare Clinical Course of Seronegative Pulmonary-Renal Syndrome. Case Reports in Critical Care, 2016, Article ID: 4893496. https://doi.org/10.1155/2016/4893496

[22] Saladi, L., Shaikh, D., Saad, M., Cancio-Rodriguez, E., et al. (2018) Renal Syndrome: A Case Report of Diffuse Alveolar Hemorrhage in Association with ANCA Negative Pauci-Immune Glomerulonephritis. Medicine, 97, e10954.

https://doi.org/10.1097/MD.0000000000010954

[23] Klemmer, P., Chalermskulrat, W., Reif, M., Hogan, S., Henke, D. and Falk, R. (2003) Plasmapheresis Therapy for Diffuse Alveolar Hemorrhage in Patients with Small-Vessel Vasculitis. American Journal of Kidney Diseases, 42, 1149-1153. https://doi.org/10.1053/j.ajkd.2003.08.015 